\title{
Differential expression of AtWAKL10 in response to nitric oxide suggests a putative role in biotic and abiotic stress responses
}

\author{
Phearom Bot ${ }^{\text {Equal first author, } 1}$, Bong Gyu Mun ${ }^{\text {Equal first author, } 1}{ }^{1}$, Qari Muhammad Imran ${ }^{1}$, Adil Hussain ${ }^{2}$, Sang Uk Lee ${ }^{1}$, Gary J \\ Loake $^{\text {Corresp., } 3}$, Bwyung-Wook Yun ${ }^{\text {Corresp. } 1}$ \\ ${ }^{1}$ Department of applied biosciences, Kyungpook National University, Daegu, Daegu, South Korea \\ 2 Department of Agriculture, Abdul Wali Khan university Mardan KPK Paksitan, Mardan, Pakistan \\ 3 Institute of Molecular Plant Sciences, University of Edinburgh, Edinburgh, United Kingdom \\ Corresponding Authors: Gary J Loake, Bwyung-Wook Yun \\ Email address: g.Loake@ed.ac.uk, bwyun@knu.ac.kr
}

Plant

defense against pathogens and abiotic stresses is regulated differentially by communicating signal transduction pathways in which nitric oxide (NO) plays a key role. Here, we show the biological

role of Arabidopsis thaliana wall-associated kinase (AtWAK) Like10 ( AtWAKL10) that exhibits greater than a

100-fold change in transcript accumulation in response to the nitric oxide (NO) donor S-nitroso-L-cysteine (CysNO), identified from high throughput RNA-seq based transcriptome analysis. Loss of AtWAKL10

function showed similar phenotype to wild type (WT) however with less

branching. The growth of atwakl10 on media supplemented with

oxidative or nitrosative stress resulted in differential results with improved

growth following treatment with CysNO but reduced growth in response to $S$ -

nitrosoglutatione

(GSNO) and methyl- viologen . Further, atwakl10 plants exhibited increased

susceptibility to virulent Pseudomonas syringae pv

tomato ( Pst ) DC3000 with a significant increase in pathogen growth and decrease in $P R 1$ transcript accumulation

compared to WT overtime. Similar results were found in response to Pst DC3000 avrB, resulting in increased

cell death as shown by increased electrolyte leakage in atwakl10. Furthermore, atwakl10 also showed increased ROS accumulation following Pst DC3000 avrB inoculation. Promoter analysis of AtWAKL10 showed transcription factor 
binding sites for biotic and abiotic stress-related

transcription factors. Further investigation into the role of AtWAKL10 in abiotic stresses showed that

following two weeks water-withholding drought condition most of the atwakl10

plants got wilted, however, the majority $(60 \%)$ of these plants recovered following re-watering. In contrast, in

response to salinity stress, atwakl10 showed reduced germination

under $150 \mathrm{mM}$ salt stress compared to WT suggesting that NO-induced AtWAKL10

differentially regulates

different abiotic stresses. Taken together, this study further elucidates the importance of NO-induced changes in gene expression and their role in plant biotic and abiotic stress tolerance. 


\section{Differential expression of AtWAKL10 in response to}

2 nitric oxide suggests a putative role in biotic and

3 abiotic stress responses

4

5

6

7

8

9

10

11

12

13

14

15

16

17

Phearom Bot ${ }^{1 a}$, Bong-Gyu Mun ${ }^{1 a}$, Qari Muhammad Imran ${ }^{1}$, Adil Hussain², Sang Uk Lee ${ }^{1}$, Gary J. Loake ${ }^{3 *}$ and Byung-Wook Yun ${ }^{1 *}$

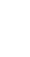

${ }^{1}$ Laboratory of Plant Functional Genomics, School of Applied Biosciences, Kyungpook National University, Daegu, Republic of Korea

${ }^{2}$ Department of agriculture Abdul Wali Khan University Mardan KPK Pakistan

${ }^{3}$ Institute of Molecular Plant Sciences, University of Edinburgh, King's Buildings, Edinburgh, UK

Corresponding Authors:

Byung-Wook yun ${ }^{1}$

702-701, Buk-gu, Daegu, 41566, Republic of Korea

Email address: bwyun@knu.ac.kr

Gary J. Loake

Institute of Molecular Plant Sciences, University of Edinburgh, King's Buildings, Edinburgh, UK Email address: g.Loake@ed.ac.uk

\section{Abstract}

Plant defense against pathogens and abiotic stresses is regulated differentially by communicating signal transduction pathways in which nitric oxide (NO) plays a key role. Here, we show the biological role of Arabidopsis thaliana wall-associated kinase (AtWAK) Like10 (AtWAKL10) that exhibits greater than a 100-fold change in transcript accumulation in response to the nitric oxide (NO) donor S-nitroso-L-cysteine (CysNO), identified from high throughput RNA-seq based transcriptome analysis. Loss of AtWAKL10 function showed similar phenotype to wild type (WT) however with less branching. The growth of atwakl10 on media supplemented with oxidative or nitrosative stress resulted in differential results with improved growth following treatment with CysNO but reduced growth in response to $S$-nitrosoglutatione (GSNO) and methyl-viologen. Further, atwakl10 plants exhibited increased susceptibility to virulent Pseudomonas syringae pv tomato (Pst) DC3000 with a significant increase in pathogen growth and decrease in $P R 1$ transcript accumulation compared to WT overtime. Similar results were found in response to Pst DC3000 avrB, resulting in increased cell death as shown by increased electrolyte leakage in atwakl10. Furthermore, atwakl10 also showed increased ROS accumulation following Pst DC3000 avrB inoculation. Promoter analysis of AtWAKL10 showed transcription factor binding sites for biotic and abiotic stress-related transcription factors. Further 
40 investigation into the role of AtWAKL10 in abiotic stresses showed that following two weeks 41 water-withholding drought condition most of the atwakl10 plants got wilted, however, the 42 majority (60\%) of these plants recovered following re-watering. In contrast, in response to 43 salinity stress, atwakl10 showed reduced germination under $150 \mathrm{mM}$ salt stress compared to WT 44 suggesting that NO-induced $A t W A K L 10$ differentially regulates different abiotic stresses. Taken 45 together, this study further elucidates the importance of NO-induced changes in gene expression 46 and their role in plant biotic and abiotic stress tolerance.

\section{Keywords:}

48 AtWAKL10, basal defense, effector-triggered immunity, promoter analysis, drought, Salt stress,

49

50

51

52

53

54

55

56

57

58

59

60

61

62

63

64

65

66

67

68

69

70

71

72

73

74

75

76

77

78

\section{Introduction}

Nitric oxide (NO), diatomic molecules is a gaseous free radical known for its signaling role under stress condition (Delledonne et al. 1998). This small redox active molecule got fair attention of scientists in the recent past not only due to its signaling but also its regulatory role. Initially identified in mammals with medical roles in cardiac diseases (Hiraga et al. 1998; Liu et al. 2004; Fraccarollo et al. 2009(Liu et al. 2004); NO was reported to have equally important roles in plants (Delledonne et al. 1998; Durner et al. 1998; Delledonne 2005). In the last couple of decades, $\mathrm{NO}$ has been reported to regulate a number of physiological processes such as germination (Beligni \& Lamattina 2000) Chlorophyll contents (Leshem et al. 1997), adventitious roots development (Pagnussat et al. 2002), root elongation (Gouvea et al. 1997), flowering time (He et al. 2004) apical dominance (Lee et al. 2008) hormonal balance (Yu et al. 2014) and plant defense (Delledonne et al. 1998; Feechan et al. 2005; Yun et al. 2011) etc.

$\mathrm{NO}$ in the presence of oxygen form different important oxides like $\mathrm{NO}_{2}$, that may further react with cellular amines and thiols. It can also react with superoxide anion radical ( $\left.{ }^{\circ}\right)$ producing such ions that later on cause significant damage to cell structure (Wendehenne et al. 2001). These molecules are also called reactive nitrogen species (RNS). The production of reactive oxygen and nitrogen species (ROS and RNS) is an important outcome in response to biotic and abiotic stress (Garcia-Mata \& Lamattina 2002; Burniston \& Wilson 2008). Controlled ROS production is extremely important for activating downstream processes. For example, hydrogen peroxide $\left(\mathrm{H}_{2} \mathrm{O}_{2}\right)$ leads to modulation of the cellular redox state and may regulate certain defense related processes including programmed cell death (PCD) and biotic (Harding et al. 2003; Suzuki et al. 2011) and abiotic stress responses (Rao \& Sresty 2000). However, contrary to well-studied reactive oxygen species (ROS), little is known about the RNS and their roles in plants. Plants have evolved fine-tuned mechanisms to cope with attempted pathogen ingress. One of these defense responses include the evolution of resistance $(R)$ genes to defend themselves against pathogen attacks. $R$-genes encode proteins that either recognize pathogen avirulence (Avr) proteins or their activities (Dangl \& Jones 2001; Jones \& Dangl 2006). Pathogen recognition prevents pathogen expansion via a variety of mechanisms, including localized programmed cell death which may involve signaling components analogous to animal apoptosis (Plocik et al. 2004). 
79 The intracellular secondary messenger guanosine 3', 5' - cyclic monophosphate (cGMP) has been 80 reported to be an important signaling molecule controlling a wide range of physio-molecular 81 responses in both prokaryotes and eukaryotes (Schaap 2005). In higher plants cGMP is reported 82 to be involved in signaling during various physiological processes (Newton \& Smith 2004). 83 These include biotic (Durner et al. 1998), abiotic (Pasqualini et al. 2009), and NO-dependent 84 signaling (Prado et al. 2004). After the perception of stress responses, the guanylyl cyclase (GC) 85 activates to catalyze the synthesis of cGMP from guanosine 5-triphosphate (GTP) (Schaap 86 2005). However, despite the well-explored role of cGMP in signaling, the role of its catalytic 87 88 enzyme GCs in higher plants is yet to be identified. BLAST searches have identified about 7 candidates GCs in Arabidopsis (Meier et al. 2010). One of these candidates is the Arabidopsis wall associated kinase-like 10 [(AtWAKL10) AT1G796800]. The WAK/WAKL genes are typically supposed to encode a class of receptor-like protein kinases having a transmembrane domain and a serine/threonine kinase domain in addition to an extracellular region that is closely associated with the cell wall (He et al. 1999). Reports suggested that some WAKs may be involved in pathogen defense responses. For example, AtWAK1 expression was induced after Pseudomonas syringae infection in Arabidopsis in NON-EXPRESSOR OF PR1 GENE (NPR1) dependent manner. Furthermore, AtWAKl is also reported to be involved in systemic acquired resistance (Maleck et al. 2000).

NO transfers its bioactivity predominantly through a key post-translational modification termed $S$-nitrosylation: a cysteine-based protein modification in which a NO moiety is covalently attached to a solvent exposed cysteine residue to form an $S$-nitrosothiol (Stamler et al. 1992; Khani et al. 2017). These SNOs then interact with intracellular sulfhydryl-containing molecules and are of great importance as they are more stable than NO (Leterrier et al. 2011). Among various SNOs, $S$-nitrosoglutathione (GSNO) is produced by the $S$-nitrosylation reaction of both NO with glutathione (GSH) and bears special importance since it is reported to be a mobile reservoir of NO (Stamler et al. 2001). Cellular GSNO homeostasis is controlled by the enzyme GSNO reductase (GSNOR) (Liu et al. 2001) which is functionally conserved across the animal, plant and bacterial species. Research studies involving GSNOR support its ameliorating role during GSNO-mediated nitrosative stress (Foster et al. 2009; Stamler et al. 2001). These properties make GSNOR vital enzyme for plant growth as cellular redox status is crucial to plant growth, development and environmental interactions (Malik et al. 2011). The loss-of-function mutation in GSNOR termed as atgsnor 1-3 resulted in a significant reduction in plant growth and compr omised defense response in Arabidopsis thaliana (Feechan et al. 2005; Yun et al. 2011). Microarray and RNA-seq analysis have revealed a number of key genes showing differential expression to NO or its donors (Parani et al. 2004; Begara-Morales et al. 2014). Recently, Hussain et al. (2016) demonstrated several hundred genes showing differential response to the NO donor CysNO. Similarly, 673 transcription factors including major transcription factor families showed differential expression to the same NO donor (Imran et al. 2018a). Here we 
119 show AtWAKL10 a candidate gene of NO signaling that exhibited more than a 100 -fold induction 120 (Fold change 124.7) in response to CysNO and exhibits a role in both plant defense and 121 oxidative stress for this gene product. AtWAKL10 is a positive regulator of basal defense, effector 122 triggered-immunity, and salt stress while negatively regulating drought stress.

123

\section{Materials \& Methods}

\subsection{Plant material, growth conditions and genotyping} Col-0 was used as WT, whereas atgsnor 1-3, a loss-of-function mutant of Arabidopsis $S$ nitrosoglutathione reductase1 (AtGSNOR1) was used as a susceptible control (Feechan et al. 2005). Loss of function mutant lines for WAKL10 (AT1G79680) was procured from the Arabidopsis Biological Resource Center (ABRC https://abrc.osu.edu/). Seeds were surfacesterilized using $50 \%$ bleach (commercial bleach) for about 1 2 minutes and grown on halfstrength Murashige and Skoog (MS) media (Murashige \& Skoog 1962). After a week, seedlings were transplanted in soil and grown under controlled light $\left(16-8 \mathrm{~h}\right.$.) and temperature $\left(23^{\circ} \mathrm{C} \pm\right.$ 2). atwakl10 plants were genotyped to identify homozygous lines using gene-specific forward and reverse and T-DNA border primers using PCR. The list of primers is given in Table. 1. The PCR conditions were initial denaturation at $94^{\circ} \mathrm{C}$ for 2 min followed by 30 cycles of $94^{\circ} \mathrm{C}$ for 20 sec, $58^{\circ} \mathrm{C}$ for $30 \mathrm{sec}$ and $72^{\circ} \mathrm{C}$ for $1 \mathrm{~min}$ and a final extension at $72^{\circ} \mathrm{C}$ for $5 \mathrm{~min}$. The identified homozygous lines were then used for further experiments.

\subsection{Oxidative and nitrosative stress assay}

To study the response of atwakl10 towards oxidative and nitrosative stress, plants were subjected to oxidative and nitrosative stress as described earlier (Imran et al. 2016). Briefly, for nitrosative stress conditions, sterilized Arabidopsis seeds from WT Col-0, atgsnorl-3 and atwakl10 were sown on media supplemented with $1 \mathrm{mM}$ CysNO or $1 \mathrm{mM} S$-nitrosoglutathione (GSNO). For exposure to oxidative stress, sterilized seeds from Col- 0 and mutant line were grown on media supplemented with $2 \mathrm{mM} \mathrm{H}_{2} \mathrm{O}_{2}$ or $1 \mu \mathrm{M}$ Methyl Viologen (Medina-Rivera et al.). For all treatments, plants were grown in 16 and $8 \mathrm{~h}$ light and dark conditions, respectively in triplicates at $23^{\circ} \mathrm{C} \pm 2$ temperature. The experiments was repeated twice and cotyledon development frequency (CDF) was recorded after 1 week as described by Imran et al. (2016). The term CDF was used for green developed cotyledons.

\subsection{Pathogen inoculations and cell death}

Pseudomonas syringae pv. tomato (Pst) strain DC3000 was cultured and maintained as described (Whalen et al. 1991). Briefly, virulent Pst DC30000 (concentration 0.0002 at $\mathrm{OD}_{600}$ i.e. $5 \times 10^{5}$ $\mathrm{CFU} \mathrm{mL}{ }^{-1}$ ) and avirulent $P s t$ DC30000 avrB (concentration 0.002 at $\mathrm{OD}_{600}$ i.e. $1 \times 10^{6} \mathrm{CFU} \mathrm{mL}^{-}$ ${ }^{1}$ ) were grown on LB (Luria-Bertani) media with appropriate antibiotics (Rifampicin, Kanamycin and Rifampicin respectively). The single colony was then transferred to LB broth and incubated at $28^{\circ} \mathrm{C}$ overnight with shaking. Both the bacterial strains were then harvested by centrifugation in $10 \mathrm{mM} \mathrm{MgCl}_{2}$. The inoculum thus prepared was infiltrated into the abaxial side of leaves at the 
157

158

159

160

161

162

163

164

165

166

167

168

169

170

171

172

173

174

175

176

177

178

179

180

181

182

183

184

185

186

187

188

189

190

191

192

193

indicated concentration. After 2 and 4 days of inoculation, disease symptoms were recorded and leaf samples (4 replicates) were harvested for bacterial growth analysis (Feechan et al. 2005).

\subsection{Histological staining}

The avirulent strain of Pst DC3000 expressing the $a v r B$ effector protein interacts with plant Rgenes thereby triggering hypersensitive cell death response (Leister et al. 1996). To see pathogen-induced cell death in vivo, all the genotypes were infiltrated with Pst DC3000 avrB and cell death in inoculated leaves was measured by trypan blue staining (Yun et al. 2011). To visualize $\mathrm{H}_{2} \mathrm{O}_{2}$ in situ we used the already established method (Yun et al. 2003). Briefly, leaves inoculated with Pst DC3000 avrB were stained with 3-3'-diaminobenzidine (DAB) which is absorbed by the plant and polymerized locally in the presence of $\mathrm{H}_{2} \mathrm{O}_{2}$ and peroxidases giving a visible brown stain. The inoculated leaves were dipped in DAB solution and put on a shaker overnight. The DAB solution was then replaced with $96 \%$ ethanol and boiled for $10 \mathrm{~min}$. The leaves were then allowed to cool-down and washed with $\mathrm{ddH}_{2} \mathrm{O}$ two times. The leaves were then mounted on microscopic slides.

\subsection{Electrolyte leakage}

Pathogen-induced cell death was quantified by measuring electrolyte leakage. Briefly, $1 \mathrm{~cm}^{2}$ leaf discs form the leaves inoculated with avirulent Pst DC3000 at concentration of $1 \times 10^{8} \mathrm{CFU} \mathrm{mL}^{-}$

${ }^{1}$ were collected in triplicates with three leaf discs per replicate. The leaf discs were washed with deionized water and put in a multi-well plate (SPL life sciences Korea) containing an equal amount of de-ionized water. Electrolyte leakage in each well was measured over time by conductivity meter (Horiba Twin Cond, Conductivity meter B-173).

\subsection{Salt and drought stress}

To test atwakl10 response to salt stress, seeds were placed on $1 / 2$ MS media in petri dishes supplemented with $100 \mathrm{mM}$ and $150 \mathrm{mM}$ salt $(\mathrm{NaCl})$. The control plants were grown on $1 / 2 \mathrm{MS}$ media only. The petri plates were placed in the growth chamber at $23^{\circ} \mathrm{C} \pm 2$ temperature for 16 and $8 \mathrm{~h}$ of light and dark period respectively. One week later, CDF was measured and pictures were taken. The drought stress was induced by withholding water for two weeks. The control plants were watered regularly. After 2 weeks, plants were examined and re-watered to see the recovery. The recovery rate was calculated as the number of recovered plants after re-watering to the number of total plants subjected to drought stress for each genotype.

\subsection{Quantitative real-time (qRT) PCR}

RNA extraction and qRT-PCR was performed as mentioned earlier. Briefly, total RNA was extracted from fresh leaf tissues using the Qiagen RNeasy Plant mini kit (Qiagen Korea). After treatment with DNase I, $1 \mu \mathrm{g}$ of total RNA was used for the synthesis of the first strand of cDNA using Omniscript RT kit and oligo dT. The cDNA was then used as a template for running RTPCR. Real-time PCR was performed in 48-well plate with Eco Real-time PCR System (Eco ${ }^{\mathrm{TM}}$ Illumina United States). In each reaction, $400 \mathrm{ng}$ of the template cDNA, $100 \mathrm{nM}$ of forward 
194

195

196

197

198

199

200

201

202

203

204

205

206

207

208

209

210

211

212

213

214

215

216

217

218

219

220

221

222

223

224

225

226

227

228

primer and reverse primer, $10 \mu \mathrm{l}$ of SYBR green master mix was used in a total of $20 \mu 1$ reaction volume. Pathogenesis-related gene $1(P R 1)$, expression was studied relative to actin expression. The primer list is given in Table. 1 .

\subsection{Promoter analysis of AtWAKL10}

Promoter sequence $1.5 \mathrm{~kb}$ upstream of the transcription initiation site was analyzed using PlantPAN 2.0 (Chow et al. 2015). Briefly, accession number of AtWAKL10 was used to search cis-regulatory elements in the promoter using Arabidopsis thaliana weight matrix. The upstream and downstream coordinates of promoter region were X: 1500, Y: 100, Z: 500. The Arabidopsis and Rice was selected as an option for identifying conserved regions. After execution of the analysis, transcription factors (TFs) and their binding sites within the promoter region were visualized by selecting few key transcription factors. Furthermore, the conserved regions among the promoter of $A t W A K L 10$ and its orthologs in another model plant rice were studied using Cross-species tool of PlantPAN.

\subsection{Statistical analysis}

For all the experiments, the data were analyzed for significance and $p$-value was determined using a two-tailed student's $t$ test using Microsoft Excel. The level of significance was set at $p \leq$ 0.05 or $p \leq 0.01$. The mean and standard error (SE) were calculated and compared with control. All the experiments were repeated from two to four times and representative results are presented.

\section{Results}

\subsection{Loss of AtWAKL10 function results in differential responses to oxidative and nitrosative stress}

T-DNA insertion in AtWAKL10 was genotyped to identify homozygous lines and further confirmed by examining its expression level in WT and atwakl10 plants. No AtWAKL10 expression was detected in the mutant line (Figure 1A; Supplementary Figure S1). Cotyledon development frequency was used as a score for plant fitness. Our results suggested that the loss of function mutant, atwakl10, showed significantly higher CDF compared to WT under CysNOinduced nitrosative stress, however, in case of GSNO, there was no significant difference (Figure. 1B, C). In contrast, on MV media there was no significant difference in CDF among WT and atwakl10 plants (Figure. 1C). Interestingly, atgsnorl-3 showed significant tolerance towards MV stress (Figure 1C). Together all these data suggest that AtWAKL10 differentially regulate different stress conditions. Under normal conditions atwakl10 plants were phenotypically similar to WT in height however, atwakl10 showed fewer branches compared to WT (Figure 1D). 
229

230

231

232

233

234

235

236

237

238

239

240

241

242

243

244

245

246

247

248

249

250

251

252

253

254

255

256

257

258

259

260

261

262

263

264

265

266

267

\subsection{AtWAKL10 is required for basal defense}

To validate the possible role of $A T W A K L 10$ in basal defense, mutant lines, as well as WT were inoculated with virulent $P$ st DC3000. The atgsnor 1-3 is susceptible to virulent Pst DC3000 compared to WT (Feechan et al. 2005; Yun et al. 2011). Symptoms of pathogen infection were clearly visible on infiltrated leaves in both atgnor 1-3 and atwakl10 lines (Figure 2A). The symptoms development results were also reflected in pathogenicity assay. We observed that at 0 day post inoculation (dpi), only atgsnor $1-3$ showed significant $(\mathrm{p} \leq 0.001)$ increase in pathogen growth while no significant difference was found in atwakl10 mutants compared to WT (Figure 2B). However, both atwakl10 and atgsnor 1-3 mutant showed significant increase in bacterial growth compared to WT at 2 and 4 dpi (Figure 2B). We hypothesized that the increased pathogen susceptibility observed in the atwakl10 line may be due to down-regulation of the salicylic acid (SA) signaling pathway, therefore we tested Pathogenesis-related 1 (PRI) gene expression which is a marker for SA-dependent gene expression. PR1 showed significantly reduced $(p \leq 0.05)$ transcript accumulation in both atgsnor $1-3$ and atwakl10 plants overtime compared to WT (Figure 2C). At 0 h post inoculation atwakl10 however showed increased $P R$ accumulation. This suggests that the susceptible phenotype of atwakl10 may be due to downregulation of SA-dependent $P R$ genes.

\subsection{AtWAKL10 positively regulates effector-triggered immunity (ETI)}

To study a possible role of AtWAKL10 in effector-triggered immunity (ETI), plants were infiltrated with avirulent PstDC3000 expressing $a v r B$. A key defense response to restrict pathogen growth in resistant plants is the hypersensitive response (HR). Therefore, we sought to determine the pathogen-induced HR response in both WT and atwakl10 mutant plants using trypan blue staining (Feechan et al. 2005). Our results suggested an increased cell death phenotype in both atwakl10 and atgsnor 1-3 plants compared to WT (Figure 3A). Similarly, pathogen growth assay showed a significant $(p \leq 0.05$ and $p \leq 0.001$ respectively) increase in number of bacterial colonies in both atwakl10 and atgsnor 1-3 plants compared to WT plants at 2 and 4 days after inoculation (Figure 3B); though, there was no significant difference in bacterial growth at 0 dpi among all genotypes. We further suggested that the increased HR response in atwakl10 plants might be due to increased ROS accumulation. Therefore, we studied hydrogen peroxide $\left(\mathrm{H}_{2} \mathrm{O}_{2}\right)$ accumulation after Pst DC3000 avrB inoculation using DAB staining. Our results suggested increased $\mathrm{H}_{2} \mathrm{O}_{2}$ accumulation in atwakl10 compared to WT (Figure 3C). HR response is often characterized by cell death at the site of infection. Therefore to further confirm the pathogen-induced cell death phenotype of atwakl10, electrolyte leakage was measured overtime following PstDC3000 avrB challenge. The atwakl10 plants showed more electrolyte leakage overtime compared to other genotypes studied; followed by atgsnor 1-3 (Figure 3D).

\subsection{Promoter of AtWAKL10 rich in binding sites for stress response transcriptional regulators}

The gene structure is crucial to study the function of a gene and plays a key role in adaptation and evolution. We, therefore, analyzed the gene structure of AtWAKL10 and found that it 
268

269

270

271

272

273

274

275

276

277

278

279

280

281

282

283

284

285

286

287

288

289

290

291

292

293

294

295

296

297

298

299

300

301

302

303

304

305

306

consists of three exons and two intronic regions (Figure 4A). Promoter region upstream of transcription initiation site regulates mechanistic control of transcription initiation by possessing sites for binding transcription factors (Palmieri et al. 2008; Imran et al. 2018a). These sites also called cis-regulatory elements regulate several cellular processes. We, therefore, analyzed the promoter of AtWAKL10 $1.5 \mathrm{~kb}$ upstream of the transcription initiation site to identify cisregulatory elements. A number of key cis-regulatory elements were identified in the promoter region (complete list given as Supplementary Table S1). Some of them were mentioned in Figure 4B including binding sites for Arabidopsis thaliana MERISTEM LAYER1 (ATML1) a transcription factor which is expressed in the L1 layer and is supposed to suggest the mechanism for cell fate specification (Abe et al. 2001). Among others were AtDREB2 involved in regulation of dehydration and high salinity responsive gene expression (Nakashima et al. 2000) and AtDREB 19 that enhanced plant performance without compromising phenotype under drought salt stress (Krishnaswamy et al. 2011). Furthermore, the important w-boxes, binding sites for WRKY TFs were also present (Figure 4B). Identification of AtWAKL10 in model crop rice showed that AtWAKL10 is similar to rice OsWAKL5 (Figure 4C).

\subsection{AtWAKL10 positively regulates $\mathrm{NaCl}$-induced salt stress}

The promoter region of AtWAKL10 showed TF binding sites (TFBS) for TFs that are involved in abiotic stress responses as mentioned in section 3.4. We, therefore, tested if there is any possible role for AtWAKL10 in abiotic stress. WT and atwakl10 lines were subjected to 100 and $150 \mathrm{mM}$ $\mathrm{NaCl}$ solution and the germination percentage was recorded after one week. Results suggested that at $100 \mathrm{mM}$ salt solution there was no significant difference in germination percentage of atwakl10 and WT plants, whereas, germination was significantly reduced at $150 \mathrm{mM}$ salt treatment [(from $100 \%$ in control media to $54 \%$ in $150 \mathrm{mM}$ ) Figure $5 \mathrm{~A}, \mathrm{~B})]$.

\section{6. atwak/10 plants exhibit increased drought tolerance}

Functional characterization of downstream NO-regulated genes revealed that they play significant roles in drought stress responses (Shi et al. 2014). Furthermore, the promoter of AtWAKL10 was having TFBS for DREB19 and DREB2. DREB2 is reportedly involved in drought tolerance (Liu et al. 1998). We, therefore, investigated the response of atwakl10 plants to drought stress. Thus, wild type and atwakl10 lines were exposed to drought through water-withholding for two weeks (Figure 6A, B). Our results indicated that atwakl10 plants exhibited improved drought tolerance compared to WT (Figure 6C). Most of the atwakl10 plants exhibited reduced wilting and recovered quickly after re-watering. In contrast, few WT plants survived the drought treatment. The recovery rate for atwakl10 plants was the highest (60\%) compared to WT (0\%) (Figure 6D).

\section{Discussion}

Most biotic and abiotic stresses have a common feature: the production of oxidative and nitrosative burst and an associated change in cellular redox potential. Exposure to RNI and ROS leads to alterations in plant redox homeostasis and can even result in cell death. Therefore, it is 
307 of paramount importance to explore the role of NO in the regulation of genes involved in key 308 plant processes. Recently, we have reported more than 6000 genes thtat showed significant 309 differential expression in response to the NO donor CysNO (Hussain et al. 2016). Among those, 310 AtWAKL10 was one candidate gene that showed more than 124-fold induction suggesting 311 AtWAKL10 might be linked to NO signaling during biotic and/or abiotic stress conditions. The 312 present investigation strongly supports a role for NO in the regulation of plant defense responses 313 to biotic and abiotic factors. WAKL10 encodes a guanyl cyclase, an enzyme that catalyzes the 314 synthesis of cGMP from GTP (Schaap 2005). In animals, cGMP stimulates biosynthesis of 315 cyclic ADP-ribose and thereby serves as a further downstream messenger of NO. cGMP levels 316 are modulated by NO in animal cells and equilibrium concentrations of cGMP are dependent on 317 NO activated guanylate cyclases (Ignarro, 2000). Our results suggested that atwakl10 plants 318 showed enhanced tolerance to the NO donor CysNO but not GSNO (Figure 1C). However, on 319 both NO donors-mediated nitrosative stress conditions, the atwakl10 plants showed reduced 320 321

322 growth and albino phenotype with more severe on GSNO-mediated nitrosative stress compared to WT. This might be because of the possible role of AtWAKL10 in RNS scavenging which may be due to its many fold induction $(>124)$ in response to CysNO. Therefore a perturbation in the 323 function may result more nitrosative stress which ultimately results in compromised plant growth

324 325

326 327 328 329

330

331

332

333

334

335

336

337

338

339

340

341

342

343

344

345

346 as suggested by many reports (Corpas \& Palma 2018; Lee et al. 2008; Lindermayr 2017). The difference in response of atwakl10 plants to different NO donors might be because of the differential accumulation of intracellular NO due to different NO release rates of NO donors (He \& Frost 2016). However, GSNO being biologically active molecule present in the cell that serves a stable reservoir of NO having the potential to modify different proteins (Frungillo et al. 2014). Further detail investigation is required to unravel the underlying mechanism of how NO regulates growth responses during nitrosative stress condition. On MV-induced oxidative stress media, both WT and atwakl10 showed reduced CDF while atgsnor 1-3 showed increased tolerance with about $80 \% \mathrm{CDF}$ percentage. The enhanced growth performance by atgsnor $1-3$ on MV media might be due to a strong antioxidant system in atgsnor1-3 (Kovacs et al. 2016). We further studied the role of AtWAKL10 in basal defense and found that atwakl10 plants were more susceptible to virulent Pst DC3000, suggesting that AtWAKL10 positively regulates basal defense (Figure 2A, B). Previous studies on $A t W A K$ genes have suggested their possible role in pathogen defense responses. In Arabidopsis, AtWAKl was induced by $P$. syringae as well as by exogenous SA application (He et al. 1998). In addition, AtWAK1 transcription is up-regulated by systemic acquired resistance (SAR) inducing conditions (Maleck et al. 2000).

WAK / WAKL proteins comprise a transmembrane domain, a cytoplasmic serine/ threonine kinases domain and an extracellular calcium binding domain (Meier et al. 2010). The intracellular messenger cGMP is an important signaling molecule in biotic stress responses in plants. Ludidi \& Gehring (2003) showed in vitro generation of cGMP from a GC motif present in an intracellular domain of the AtWAKL10 protein. WAKL has a well-documented role in regulating SA-dependent defense response (He et al. 1998). Few WAKL genes have been implicated in SA/JA cross talk. The susceptibility of atwakl10 to Pst DC3000 has indicated the 
347 role of WAKL10 in defense signaling (Figure $2 \mathrm{~A}, \mathrm{~B}$ ). Expression dynamics of SA responsive 348 genes has provided evidence for the involvement of WAKL10 gene in SA responses. Further, the

349

350

351

352

353

354

355

356

357

358

359

360

361

362

363

364

365

366

367

368

369

370

371

372

373

374

375

376

377

378

379

380

381

382

383

384

385

386 promoter region of Arabidopsis $A t W A K$ and $A t W A K L$ genes harbor binding sites for WRKY transcription factors (Meier et al. 2010). These reports are in accordance with our results in which we also found WRKY TFBS in the promoter region of AtWAKL10. This TF family has been extensively studied for its role in regulating the expression of defense response genes (Eulgem et al. 1999). Li et al. (2004) described the role of Arabidopsis WRKY70 as a node of convergence between SA and JA signaling. They reported overexpression of WRKY70 caused activation of SA-induced $P R$ genes and suppression of JA responsive $P D F 1.2$ gene (Meier et al. 2010). Recently we have reported a member of the WRKY TF family that was regulated at the transcriptional level in response to NO, in an RNA-seq mediated transcriptome analysis (Imran et al. 2018b). To study the role of AtWAKL10 in effector-triggered immunity, we inoculated plants with Pst DC3000 expressing avrB effector. The ultimate response of effector- triggered immunity as a result of interaction between avr and $R$ gene products is hypersensitive cell death response (HR) (Jones \& Dangl 2006). Though the exact mechanisms of $R$-avr interactions are still being investigated, the requirement of salicylic acid (SA) has been shown in many plantpathogen interactions (MauchMani \& Slusarenko 1996). Therefore, we quantified the HR response by histological staining using trypan blue. We found that atwakl10 lines showed an enhanced HR response (Figure 3A). The HR is mostly associated with resistance to Biotrophic pathogens; however, atwakl10 plants showed a significantly greater bacterial titer compared to WT (Figure 2B; 3B). Similarly, the atgsnor 1-3 mutant line also showed increased susceptibility to virulent and avirulent Pst DC3000 with increased CFU counts (Figure 2B, 3B). These results are in accordance with Feechan et al. (2005) and who for the first time reported that perturbation in GSNORI function results in compromised defense response. One of the possible explanation for this could be the hemibiotrophic nature of $P$ st which even can feed and survive on dead matter and multiply as suggested by (Lee \& Rose 2010). Secondly, after attempted pathogen infection, the production of ROS such as superoxide $\left(\mathrm{O}_{2}{ }^{-}\right)$and hydrogen peroxide $\left(\mathrm{H}_{2} \mathrm{O}_{2}\right)$ is one of the early events to occur. These ROS are either helping in strengthening the cell wall thereby trying to restrict pathogen invasion (Bradley et al. 1992) or act as a signaling molecule to induce defense response such as a rapid HR response (Alvarez et al. 1998). Therefore, we suggest that the induced $\mathrm{HR}$ response in atwakl10 might be due to increased pathogen-induced $\mathrm{H}_{2} \mathrm{O}_{2}$ accumulation. We, therefore, sought to determine the accumulation of $\mathrm{H}_{2} \mathrm{O}_{2}$ in WT and $\mathrm{KO}$ mutant lines. Our results revealed an increased accumulation of $\mathrm{H}_{2} \mathrm{O}_{2}$ in atwakl10 mutant line supporting our hypothesis (Figure 3C).

Promoter analysis of AtWAKL10 revealed the presence of key TFBS that are involved in abiotic stress tolerance (Figure 4B). We, therefore, tested atwakl10 plants for their possible response to salt and drought stress. The atwakl10 line showed a differential response to both stresses. In the case of salt stress, atwakl10 plants showed a reduced germination percentage at $150 \mathrm{mM}$ (Figure 5A, B). Literature reports have suggested that cGMP maintains a lower $\mathrm{Na}\left({ }^{+}\right) / \mathrm{K}\left({ }^{+}\right)$ratio and increasing the plasma membrane $\mathrm{H}(+)$ ATPase gene expression that reduces the injury caused by 
387

388

389

390

391

392

393

394

395

396

397

398

399

400

401

402

403

404

405

406

407

408

409

410

411

412

413

414

415

416

417

418

419

420

421

422

423

424

425

salt stress (Li et al. 2011). AtWAKL10 that encodes GC and catalyzes the synthesis of cGMP might, therefore, be important in regulating salt stress positively. Contrary to this, in response to drought stress atwakl10 plants showed a higher survival rate (Figure 6C, D). The promoter of AtWAKL10 has TFBS for mostly drought-responsive genes such as DREB19, DREB2 (Liu et al. 1998) and ABA-responsive WRKY18 and WRKY40 (Figure 4B). Previous reports have explored the role of DREBs in the regulation of abiotic stress responses including drought (Lata \& Prasad 2011). Similarly, Chen et al. (2010) reported the role of WRKY18 and WRKY40 in abscisic acid and abiotic stress responses in Arabidopsis. Transcription factors are regulatory genes that mediate the expression of genes in response to a particular stimulus. Therefore, the presence of TFBS related to drought suggests regulation of AtWAKL10 by drought-related TFs; however, the exact mechanism needs to be explored further.

\section{Conclusions}

AtWAKL10 one of the candidate genes for NO signaling showed 124.7 fold change in response to CysNO in RNA-seq based transcriptomic study (Hussain et al. 2016). Investigation into the role of $A t W A K L 10$ in plant biology using functional genomics revealed that loss of function in AtWAKL10 resulted in no significant changes in CDF under control and oxidative stress condition whereas under CysNO mediated nitrosative stress condition it showed a significant increase in CDF compared to WT. Furthermore, AtWAKL10 showed positive regulation of basal and $R$-gene mediated resistance. Promoter analysis of AtWAKL10 suggested its role in abiotic stresses. We observed that AtWAKL10 positively regulates salt while negatively regulates drought stress. However, further investigation is required for dissecting the underlying pathways that combine or separates biotic and abiotic stress responses.

\section{Acknowledgement}

We are thankful to Arabidopsis biological resource center (ABRC) and The European Arabidopsis Stock Center for providing us seeds. We also extend our gratitude to The Arabidopsis information resource (TAIR) for providing useful information about the Gene/Protein function.

\section{References}

Abe M, Takahashi T, and Komeda Y. 2001. Identification of a cis-regulatory element for L1 layer-specific gene expression, which is targeted by an L1-specific homeodomain protein. The Plant Journal 26:487-494.

Alvarez ME, Pennell RI, Meijer PJ, Ishikawa A, Dixon RA, and Lamb C. 1998. Reactive oxygen intermediates mediate a systemic signal network in the establishment of plant immunity. 
426

427

428

429

430

431

432

433

434

435

436

437

438

439

440

441

442

443

444

445

446

447

448

449

450

451

452

453

454

455

456

457

458

459

460

461

462

463

464

465

466

467

468

469

470

471

Cell 92:773-784. Doi 10.1016/S0092-8674(00)81405-1

Begara-Morales JC, Sánchez-Calvo B, Luque F, Leyva-Pérez MO, Leterrier M, Corpas FJ, and Barroso JB. 2014. Differential transcriptomic analysis by RNA-Seq of GSNO-responsive genes between Arabidopsis roots and leaves. Plant and Cell Physiology:pcu044.

Beligni MV, and Lamattina L. 2000. Nitric oxide stimulates seed germination and de-etiolation, and inhibits hypocotyl elongation, three light-inducible responses in plants. Planta 210:215-221. Doi 10.1007/P100008128

Bradley DJ, Kjellbom P, and Lamb CJ. 1992. Elicitor-and wound-induced oxidative crosslinking of a proline-rich plant cell wall protein: a novel, rapid defense response. Cell 70:21-30.

Burniston MT, and Wilson DJ. 2008. Radioiodine ablation outcomes after imaging with I-123 or I-131: Is no news good news? Journal of Nuclear Medicine 49:166-166. 10.2967/jnumed.107.047076

Chen H, Lai ZB, Shi JW, Xiao Y, Chen ZX, and Xu XP. 2010. Roles of Arabidopsis WRKY18, WRKY40 and WRKY60 transcription factors in plant responses to abscisic acid and abiotic stress. Bmc Plant Biology 10. Artn 28110.1186/1471-2229-10-281

Chow C-N, Zheng H-Q, Wu N-Y, Chien C-H, Huang H-D, Lee T-Y, Chiang-Hsieh Y-F, Hou PF, Yang T-Y, and Chang W-C. 2015. PlantPAN 2.0: an update of plant promoter analysis navigator for reconstructing transcriptional regulatory networks in plants. Nucleic Acids Research 44:D1154-D1160.

Corpas FJ, and Palma JM. 2018. Assessing Nitric Oxide (NO) in Higher Plants: An Outline. Nitrogen 1:3.

Dangl JL, and Jones JDG. 2001. Plant pathogens and integrated defence responses to infection. Nature 411:826-833. Doi 10.1038/35081161

Jones JDG, and Dangl JL. 2006. The plant immune system. Nature 444:323-329. 10.1038 /nature 05286

Delledonne M. 2005. NO news is good news for plants. Current Opinion in Plant Biology 8:390396. 10.1016/j.pbi.2005.05.002

Delledonne M, Xia Y, Dixon RA, and Lamb C. 1998. Nitric oxide functions as a signal in plant disease resistance. Nature 394:585-588.

Durner J, and Klessig DF. 1999. Nitric oxide as a signal in plants. Current Opinion in Plant Biology 2:369-374. http://dx.doi.org/10.1016/S1369-5266(99)00007-2

Durner J, Wendehenne D, and Klessig DF. 1998. Defense gene induction in tobacco by nitric oxide, cyclic GMP, and cyclic ADP-ribose. Proceedings of the National Academy of Sciences 95:10328-10333. 10.1073/pnas.95.17.10328

Eulgem T, Rushton PJ, Schmelzer E, Hahlbrock K, and Somssich IE. 1999. Early nuclear events in plant defence signalling: rapid gene activation by WRKY transcription factors. Embo Journal 18:4689-4699. DOI 10.1093/emboj/18.17.4689

Feechan A, Kwon E, Yun B-W, Wang Y, Pallas JA, and Loake GJ. 2005. A central role for Snitrosothiols in plant disease resistance. Proceedings of the National Academy of Sciences 102:8054-8059.

Foster MW, Liu LM, Zeng M, Hess DT, and Stamler JS. 2009. A Genetic Analysis of Nitrosative Stress. Biochemistry 48:792-799. 10.1021/bi801813n

Fraccarollo D, Galuppo P, Motschenbacher S, Ruetten H, Berbner A, Schaefer A, Ertl G, and Bauersachs J. 2009. NO Independent Activation of Soluble Guanylyl Cyclase Provides

Peer) reviewing PDF | (2018:12:33544:2:0:NEW 28 Jun 2019) 
472

473

474

475

476

477

478

479

480

481

482

483

484

485

486

487

488

489

490

491

492

493

494

495

496

497

498

499

500

501

502

503

504

505

506

507

508

509

510

511

512

513

514

515

516

Additional Benefit to ACE Inhibition on Cardiac Remodeling Postinfarction. Circulation 120:S852-S853.

Frungillo L, Skelly MJ, Loake GJ, Spoel SH, and Salgado I. 2014. S-nitrosothiols regulate nitric oxide production and storage in plants through the nitrogen assimilation pathway. Nature Communications 5. ARTN 5401

Garcia-Mata C, and Lamattina L. 2002. Nitric oxide and abscisic acid cross talk in guard cells. Plant Physiology 128:790-792. 10.1104/pp.011020

Gouvea CMCP, Souza JF, Magalhaes ACN, and Martins IS. 1997. NO-releasing substances that induce growth elongation in maize root segments. Plant Growth Regulation 21:183-187. Doi 10.1023/A:1005837012203

Harding HP, Zhang YH, Zeng HQ, Novoa I, Lu PD, Calfon M, Sadri N, Yun C, Popko B, Paules R, Stojdl DF, Bell JC, Hettmann T, Leiden JM, and Ron D. 2003. An integrated stress response regulates amino acid metabolism and resistance to oxidative stress. Molecular Cell 11:619-633. Doi 10.1016/S1097-2765(03)00105-9

He WL, and Frost MC. 2016. Direct measurement of actual levels of nitric oxide (NO) in cell culture conditions using soluble NO donors. Redox Biology 9:1-14.

10.1016/j.redox.2016.05.002

He Y, Tang R-H, Hao Y, Stevens RD, Cook CW, Ahn SM, Jing L, Yang Z, Chen L, Guo F, Fiorani F, Jackson RB, Crawford NM, and Pei Z-M. 2004. Nitric Oxide Represses the Arabidopsis Floral Transition. Science 305:1968-1971. 10.1126/science.1098837

He ZH, Cheeseman I, He DZ, and Kohorn BD. 1999. A cluster of five cell wall-associated receptor kinase genes, Wak1-5, are expressed in specific organs of Arabidopsis. Plant molecular biology 39:1189-1196. Doi 10.1023/A:1006197318246

He ZH, He DZ, and Kohorn BD. 1998. Requirement for the induced expression of a cell wall associated receptor kinase for survival during the pathogen response. Plant Journal 14:55-63. DOI 10.1046/j.1365-313X.1998.00092.x

Hiraga H, Okubo T, Suto N, Sato K, Yamamoto T, Yoshida I, and Okumura K. 1998. Nitric oxide (NO) attenuates cardiac contractility assessed by Emax in the dog heart: A study with a spontaneous NO donor. Circulation 98:257-257.

Hussain A, Mun BG, Imran QM, Lee SU, Adamu TA, Shahid M, Kim KM, and Yun BW. 2016. Nitric oxide mediated transcriptome profiling reveals activation of multiple regulatory pathways in Arabidopsis thaliana. Frontiers in Plant Science 7. ARTN 975 10.3389/fpls.2016.00975

Ignarro, L. (2000). Nitric Oxide $1^{\text {st }}$ Edition. Academic Press.

Imran QM, Falak N, Hussain A, Mun B-G, Sharma A, Lee S-U, Kim K-M, and Yun B-W. 2016. Nitric oxide responsive heavy metal-associated gene AtHMAD1 contributes to development and disease resistance in Arabidopsis thaliana. Frontiers in Plant Science 7. Imran QM, Hussain A, Lee S-U, Mun B-G, Falak N, Loake GJ, and Yun B-W. 2018a. Transcriptome profile of NO-induced Arabidopsis transcription factor genes suggests their putative regulatory role in multiple biological processes. Scientific Reports 8:771.

Imran QM, Hussain A, Mun BG, Lee SU, Asaf S, Ali MA, Lee IJ, and Yun BW. 2018b. Transcriptome wide identification and characterization of NO-responsive WRKY transcription factors in Arabidopsis thaliana L. Environmental and Experimental Botany 148:128-143. 10.1016/j.envexpbot.2018.01.010

Jones JDG, and Dangl JL. 2006. The plant immune system. Nature 444:323-329.

Peer] reviewing PDF | (2018:12:33544:2:0:NEW 28 Jun 2019) 
517

518

519

520

521

522

523

524

525

526

527

528

529

530

531

532

533

534

535

536

537

538

539

540

541

542

543

544

545

546

547

548

549

550

551

552

553

554

555

556

557

558

559

560 10.1038/nature05286

Khani M, Davidson JA, Sosnowski MR, and Scott ES. 2017. Atypical growth of Phoma koolunga on cultural media and on plants artificially inoculated in environmentally controlled conditions. Australasian Plant Pathology 46:511-514. 10.1007/s13313-017-0515-y

Kovacs I, Holzmeister C, Wirtz M, Geerlof A, Frohlich T, Romling G, Kuruthukulangarakoola GT, Linster E, Hell R, Arnold GJ, Durner J, and Lindermayr C. 2016. ROS-Mediated Inhibition of S-nitrosoglutathione Reductase Contributes to the Activation of Antioxidative Mechanisms. Frontiers in Plant Science 7. ARTN $166910.3389 /$ fpls. 2016.01669

Krishnaswamy S, Verma S, Rahman MH, and Kav NN. 2011. Functional characterization of four APETALA2-family genes (RAP2. 6, RAP2. 6L, DREB19 and DREB26) in Arabidopsis. Plant molecular biology 75:107-127.

Lata C, and Prasad M. 2011. Role of DREBs in regulation of abiotic stress responses in plants. Journal of Experimental Botany 62:4731-4748. 10.1093/jxb/err210

Lee U, Wie C, Fernandez BO, Feelisch M, and Vierling E. 2008. Modulation of nitrosative stress by S-nitrosoglutathione reductase is critical for thermotolerance and plant growth in Arabidopsis. The Plant Cell Online 20:786-802. 10.1105/tpc.107.052647

Lee SJ, and Rose JK. 2010. Mediation of the transition from biotrophy to necrotrophy in hemibiotrophic plant pathogens by secreted effector proteins. Plant Signal Behav 5:769772.

Leister RT, Ausubel FM, and Katagiri F. 1996. Molecular recognition of pathogen attack occurs inside of plant cells in plant disease resistance specified by the Arabidopsis genes RPS2 and RPM1. Proceedings of the National Academy of Sciences of the United States of America 93:15497-15502. DOI 10.1073/pnas.93.26.15497

Leterrier M, Chaki M, Airaki M, Valderrama R, Palma JM, Barroso JB, and Corpas FJ. 2011. Function of S-nitrosoglutathione reductase (GSNOR) in plant development and under biotic/abiotic stress. Plant Signal Behav 6:789-793

Leshem YY, Haramaty E, Iluz D, Malik Z, Sofer Y, Roitman L, and Leshem Y. 1997. Effect of stress nitric oxide (NO): Interaction between chlorophyll fluorescence, galactolipid fluidity and lipoxygenase activity. Plant Physiology and Biochemistry 35:573-579.

Li J, Brader G, and Palva ET. 2004. The WRKY70 transcription factor: a node of convergence for jasmonate-mediated and salicylate-mediated signals in plant defense. The Plant Cell 16:319-331.

Li JS, Wang XM, Zhang YL, Jia HL, and Bi YR. 2011. cGMP regulates hydrogen peroxide accumulation in calcium-dependent salt resistance pathway in Arabidopsis thaliana roots. Planta 234:709-722. 10.1007/s00425-011-1439-3

Lindermayr C. 2017. Crosstalk between reactive oxygen species and nitric oxide in plants: Key role of S-nitrosoglutathione reductase. Free Radical Biology and Medicine.

Liu LM, Hausladen A, Zeng M, Que L, Heitman J, and Stamler JS. 2001. A metabolic enzyme for S-nitrosothiol conserved from bacteria to humans. Nature 410:490-494. Doi $10.1038 / 35068596$

Liu LM, Yan Y, Zeng M, Zhang J, Hanes MA, Ahearn G, McMahon TJ, Dickfeld T, Marshall HE, Que LG, and Stamler JS. 2004. Essential roles of S-nitrosothiols in vascular homeostasis and endotoxic shock. Nitric Oxide-Biology and Chemistry 11:39-39.

Peer) reviewing PDF | (2018:12:33544:2:0:NEW 28 Jun 2019) 
561

562

563

564

565

566

567

568

569

570

571

572

573

574

575

576

577

578

579

580

581

582

583

584

585

586

587

588

589

590

591

592

593

594

595

596

597

598

599

600

601

602

603

604

605

606

Liu Q, Kasuga M, Sakuma Y, Abe H, Miura S, Yamaguchi-Shinozaki K, and Shinozaki K. 1998. Two transcription factors, DREB1 and DREB2, with an EREBP/AP2 DNA binding domain separate two cellular signal transduction pathways in drought- and lowtemperature-responsive gene expression, respectively, in Arabidopsis. Plant Cell 10:1391-1406. DOI 10.1105/tpc.10.8.1391

Ludidi N, and Gehring C. 2003. Identification of a novel protein with guanylyl cyclase activity in Arabidopsis thaliana. Journal of Biological Chemistry 278:6490-6494. 10.1074/jbc.M210983200

Maleck K, Levine A, Eulgem T, Morgan A, Schmid J, Lawton KA, Dangl JL, and Dietrich RA. 2000. The transcriptome of Arabidopsis thaliana during systemic acquired resistance. Nature Genetics 26:403-410.

Malik SI, Hussain A, Yun BW, Spoel SH, and Loake GJ. 2011. GSNOR-mediated denitrosylation in the plant defence response. Plant Science 181:540-544. 10.1016/j.plantsci.2011.04.004

MauchMani B, and Slusarenko AJ. 1996. Production of salicylic acid precursors is a major function of phenylalanine ammonia-lyase in the resistance of arabidopsis to Peronospora parasitica. Plant Cell 8:203-212.

Medina-Rivera A, Defrance M, Sand O, Herrmann C, Castro-Mondragon JA, Delerce J, Jaeger S, Blanchet C, Vincens P, Caron C, Staines DM, Contreras-Moreira B, Artufel M, Charbonnier-Khamvongsa L, Hernandez C, Thieffry D, Thomas-Chollier M, and van Helden J. 2015. RSAT 2015: Regulatory Sequence Analysis Tools. Nucleic Acids Research 43:W50-W56. 10.1093/nar/gkv362

Meier S, Ruzvidzo O, Morse M, Donaldson L, Kwezi L, and Gehring C. 2010. The Arabidopsis Wall Associated Kinase-Like 10 Gene Encodes a Functional Guanylyl Cyclase and Is Co-Expressed with Pathogen Defense Related Genes. Plos One 5. ARTN e890410.1371/journal.pone.0008904

Murashige T, and Skoog F. 1962. A revised medium for rapid growth and bio assays with tobacco tissue cultures. Physiologia Plantarum 15:473-497.

Nakashima K, Shinwari ZK, Sakuma Y, Seki M, Miura S, Shinozaki K, and YamaguchiShinozaki K. 2000. Organization and expression of two Arabidopsis DREB2 genes encoding DRE-binding proteins involved in dehydration- and high-salinity-responsive gene expression. Plant molecular biology 42:657-665. Doi 10.1023/A:1006321900483

Newton RP, and Smith CJ. 2004. Cyclic nucleotides. Phytochemistry 65:2423-2437. 10.1016/j.phytochem.2004.07.026

Pagnussat GC, Simontacchi M, Puntarulo S, and Lamattina L. 2002. Nitric oxide is required for root organogenesis. Plant Physiology 129:954-956. 10.1104/pp.004036

Palmieri MC, Sell S, Huang X, Scherf M, Werner T, Durner J, and Lindermayr C. 2008. Nitric oxide-responsive genes and promoters in Arabidopsis thaliana: a bioinformatics approach. Journal of Experimental Botany 59:177-186. 10.1093/jxb/erm345

Parani M, Rudrabhatla S, Myers R, Weirich H, Smith B, Leaman DW, and Goldman SL. 2004. Microarray analysis of nitric oxide responsive transcripts in Arabidopsis. Plant Biotechnology Journal 2:359-366.

Pasqualini S, Meier S, Gehring C, Madeo L, Fornaciari M, Romano B, and Ederli L. 2009. Ozone and nitric oxide induce cGMP-dependent and -independent transcription of 
607

608

609

610

611

612

613

614

615

616

617

618

619

620

621

622

623

624

625

626

627

628

629

630

631

632

633

634

635

636

637

638

639

640

641

642

643

644

645

646

647

648

649

650 defence genes in tobacco. New Phytologist 181:860-870. 10.1111/j.1469-

8137.2008.02711.x

Plocik A, Layden J, and Kesseli R. 2004. Comparative analysis of NBS domain sequences of NBS-LRR disease resistance genes from sunflower, lettuce, and chicory. Molecular Phylogenetics and Evolution 31:153-163. 10.1016/S1055-7903(03)00274-4

Prado AM, Porterfield DM, and Feijo JA. 2004. Nitric oxide is involved in growth regulation and re-orientation of pollen tubes. Development 131:2707-2714. 10.1242/dev.01153

Rao KVM, and Sresty TVS. 2000. Antioxidative parameters in the seedlings of pigeonpea (Cajanus cajan (L.) Millspaugh) in response to Zn and Ni stresses. Plant Science 157:113-128.

Schaap P. 2005. Guanylyl cyclases across the tree of life. Frontiers in Bioscience-Landmark 10:1485-U1485. Doi 10.2741/1633

Shi WN, Liu DD, Hao LL, Wu CA, Guo XQ, and Li H. 2014. GhWRKY39, a member of the WRKY transcription factor family in cotton, has a positive role in disease resistance and salt stress tolerance. Plant Cell Tissue and Organ Culture 118:17-32. 10.1007/s11240-0140458-8

Stamler JS, Lamas S, and Fang FC. 2001. Nitrosylation: the prototypic redox-based signaling mechanism. Cell 106:675-683.

Stamler JS, Simon DI, Osborne JA, Mullins ME, Jaraki O, Michel T, Singel DJ, and Loscalzo J. 1992. S-nitrosylation of proteins with nitric oxide: synthesis and characterization of biologically active compounds. Proc Natl Acad Sci U S A 89:444-448.

Suzuki N, Miller G, Morales J, Shulaev V, Torres MA, and Mittler R. 2011. Respiratory burst oxidases: the engines of ROS signaling. Current Opinion in Plant Biology 14:691-699. 10.1016/j.pbi.2011.07.014

Wendehenne D, Pugin A, Klessig DF, and Durner J. 2001. Nitric oxide: comparative synthesis and signaling in animal and plant cells. Trends in Plant Science 6:177-183. 10.1016/S1360-1385(01)01893-3

Yu MD, Lamattina L, Spoel SH, and Loake GJ. 2014. Nitric oxide function in plant biology: a redox cue in deconvolution. New Phytologist 202:1142-1156. 10.1111/nph.12739

Yun B-W, Feechan A, Yin M, Saidi NBB, Le Bihan T, Yu M, Moore JW, Kang J-G, Kwon E, Spoel SH, Pallas JA, and Loake GJ. 2011. S-nitrosylation of NADPH oxidase regulates cell death in plant immunity. Nature:264-268. http://www.nature.com/nature/journal/v478/n7368/abs/nature10427.html\#supplementaryinformation

Yun BW, Atkinson HA, Gaborit C, Greenland A, Read ND, Pallas JA, and Loake GJ. 2003. Loss of actin cytoskeletal function and EDS1 activity, in combination, severely compromises non-host resistance in Arabidopsis against wheat powdery mildew. Plant Journal 34:768777. DOI 10.1046/j.1365-313X.2003.01773.x

Whalen MC, Innes RW, Bent AF, and Staskawicz BJ. 1991. Identification of Pseudomonas syringae pathogens of Arabidopsis and a bacterial locus determining avirulence on both Arabidopsis and soybean. Plant Cell 3:49-59. 10.1105/tpc.3.1.49 
651 Figure 1. The response of atwakl10 plants to oxidative and nitrosative stress.

652 (A) genotyping and expression of AtWAKL10 in WT and atwakl10 mutants (B) Germination of 653 atwakl10 and WT plants on control, oxidative (MV induced) and nitrosative stress (CysNO and 654 GSNO induced) media. (C) CDF of atwakl10 plants under indicated stressed conditions (D)

655 Phenotypes of the indicated genotypes. All the data points in panel $\mathrm{C}$ are the mean of three 656 replicates and the experiment was repeated about two times with similar results. The labels $\mathrm{F}+\mathrm{R}$ 657 in panel A denotes gene-specific forward and reverse primers while B denotes Sail left border 658 primer. Error bars represent \pm SE. significant differences are represented by asterisks (Student's

659

660

661

662

663

664

665

666

667

668

669

670

671

672

673

674

675

676

677

678

679

680

681

682

683

684

685

686

687

688

689

690

691

692

693

694

695

696 t-test) * represents $\mathrm{P}<0.05$ and $* *$ represents $\mathrm{p}<0.01$.

\section{Figure 2. AtWAKL10 negatively regulates basal defense.}

(A) Symptom development after virulent $P s t$ DC3000 inoculation. (B) Pathogen growth after virulent Pst DC3000 inoculation and (C) PR gene relative expression after attempted Pst DC3000 (virulent) inoculation. The data points are the mean of at least three replicates. The pathogenicity experiment was repeated four times with similar results. Error bars represent $\pm \mathrm{SE}$ while asterisk represents significant difference compared to WT [Student's t-test, * represents $p<0.05, * *$ represents $p<0.01$ and ${ }^{* * *}$ represents $\left.p<0.001\right]$.

\section{Figure 3. AtWAKL10 positively regulates effector-triggered immunity.}

(A) Trypan blue staining showing HR response. (B) Pathogen growth determined as log colony forming unit (CFU) (C) Diaminobenzidine (DAB) staining depicting $\mathrm{H}_{2} \mathrm{O}_{2}$ accumulation and (D) cell death-induced electrolyte leakage after Pst DC3000 avrB challenge. The background in A and $\mathrm{C}$ are modified for more clarity. The data points in $\mathrm{B}$ and $\mathrm{D}$ are the mean of three replicates. The pathogenicity experiment was repeated about 3 times while that of electrolyte leakage was repeated twice with similar results. Error bars represent $\pm \mathrm{SE}(\mathrm{n}=3)$ while significant differences compared to WT are marked with asterisks [Student's t-test * represents $p<0.05, * *$ represents $p<0.01$ and ${ }^{* * *}$ represents $\left.p<0.001\right]$.

\section{Figure 4. Promoter analysis of AtWAKL10.}

(A) Genetic structure of AtWAKL10. Green bars represent exons, while gaps between two exonic regions are introns. (B) Position and visualization of different transcription factor binding site (TFBS) $1 \mathrm{~Kb}$ upstream of transcription initiation site and (C) comparison of conserved regions of AtWAKL10 and its orthologs in rice.

Figure 5. atwakl10 plants show reduced germination percentage under high salinity conditions.
(A) Germination of atwakl10 and WT plants on different salt conditions. (B) Germination percentage in WT and mutant line after exposure to different salinity conditions. The data in the graph represents the mean of three replicates. The experiment was repeated three times with similar results. Error bars represents $\pm \mathrm{SE}(\mathrm{n}=3)$.

\section{Figure 6. atwakl10 plants exhibit drought tolerance.}

(A) Phenotypes of indicated genotypes 0 days post-drought (after withholding water). (B) After 2 weeks of drought stress. (C) Phenotypes of plants after 2 weeks of drought stress, followed by re- 
697 watering. (D) Quantification of recovery rates after re-watering. The experiment was repeated 698 twice with similar results.

699 


\section{Figure 1}

The response of atwakl10 plants to oxidative and nitrosative stress

(A) genotyping and expression of AtWAKL10 in WT and atwalk10 mutants (B) Germination of atwalk10 and WT plants on control, oxidative (MV induced) and nitrosative stress (CysNO and GSNO induced) media. (C) CDF of atwakl10 plants under indicated stressed conditions (D) Phenotypes of the indicated genotypes. All the data points in panel $\mathrm{C}$ are the mean of three replicates and the experiment was repeated about two times with similar results. The labels $F+R$ in panel $A$ denotes gene-specific forward and reverse primers while $B$ denotes Sail left border primer. Error bars represent $\pm \mathrm{SE}$. significant differences are represented by asterisks (Student's t-test with $95\left(^{*}\right)$ and $99 \%(* *)$ confidence levels. 


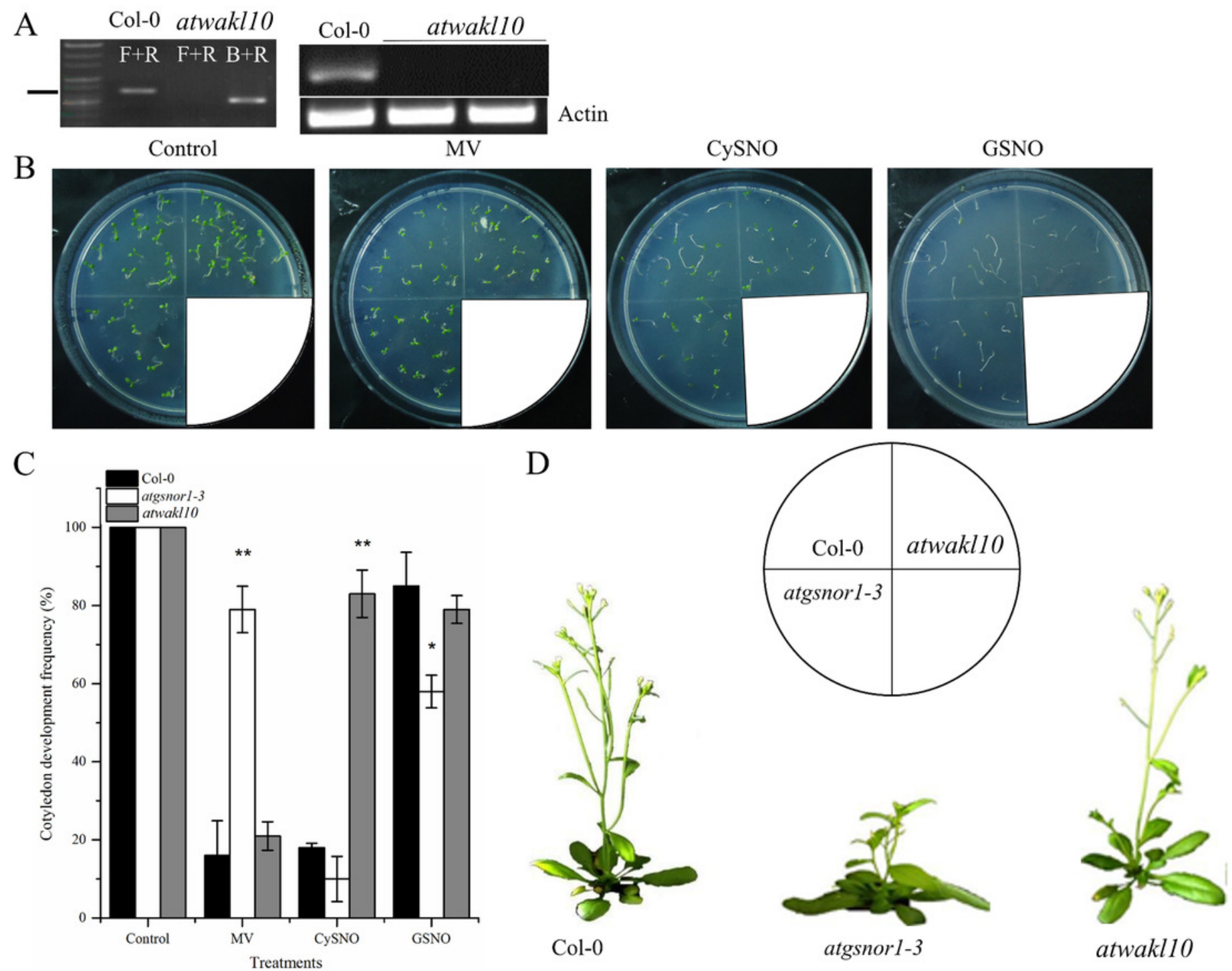


Figure 2

AtWALK10 negatively regulates basal defense

(A) Symptom development after virulent Pst DC3000 inoculation. (B) Pathogen growth after virulent Pst DC3000 inoculation and (C) PR1 gene relative expression after attempted Pst DC3000 (virulent) inoculation. The data points are the mean of at least three replicates. The pathogenicity experiment was repeated four times with similar results. Error bars represent \pm SE while asterisk represents significant difference compared to WT [Student's t-test with $95\left(^{*}\right)$ and $99 \%(* *)$ confidence levels]. 


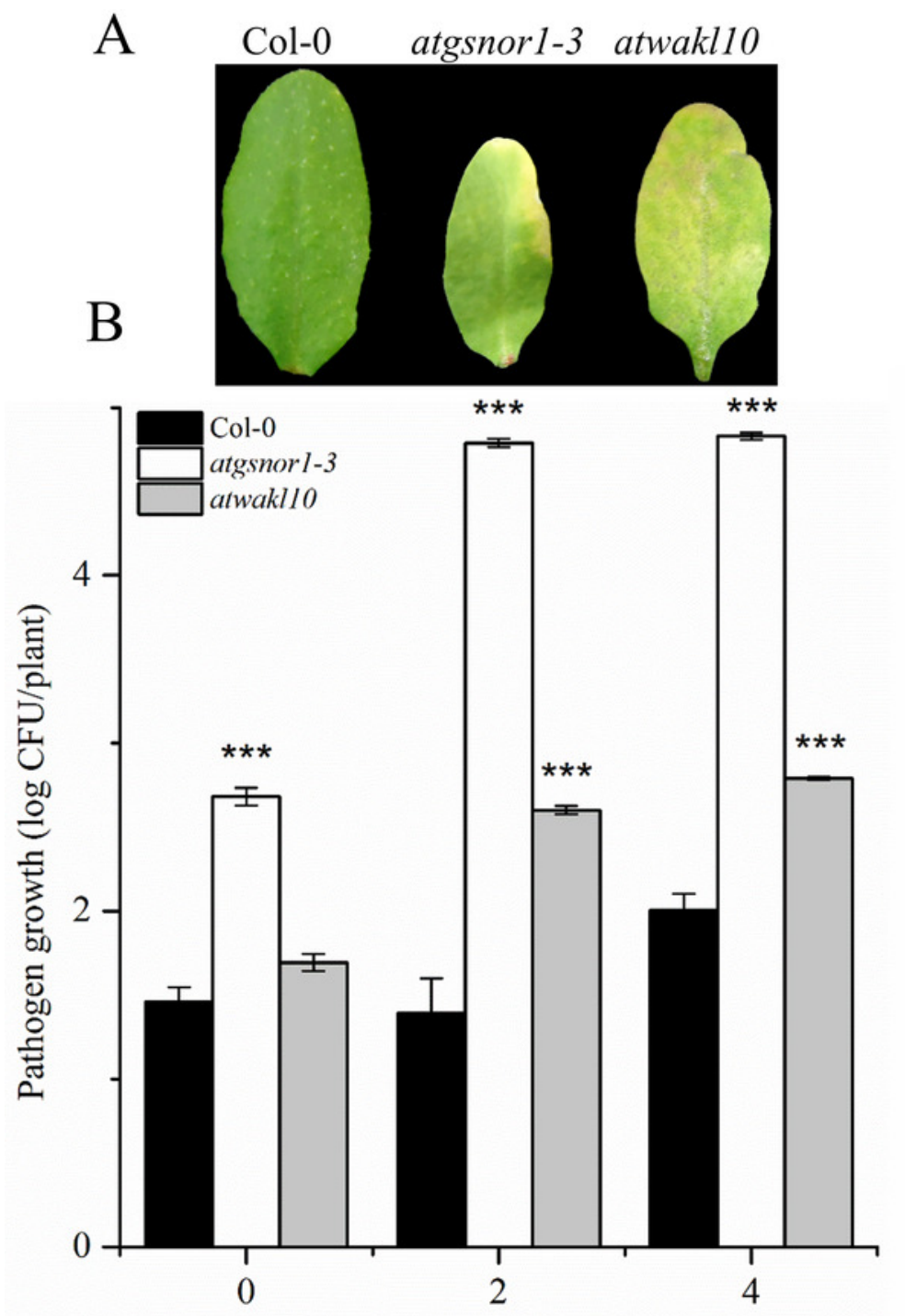

Days post inoculation (dpi)

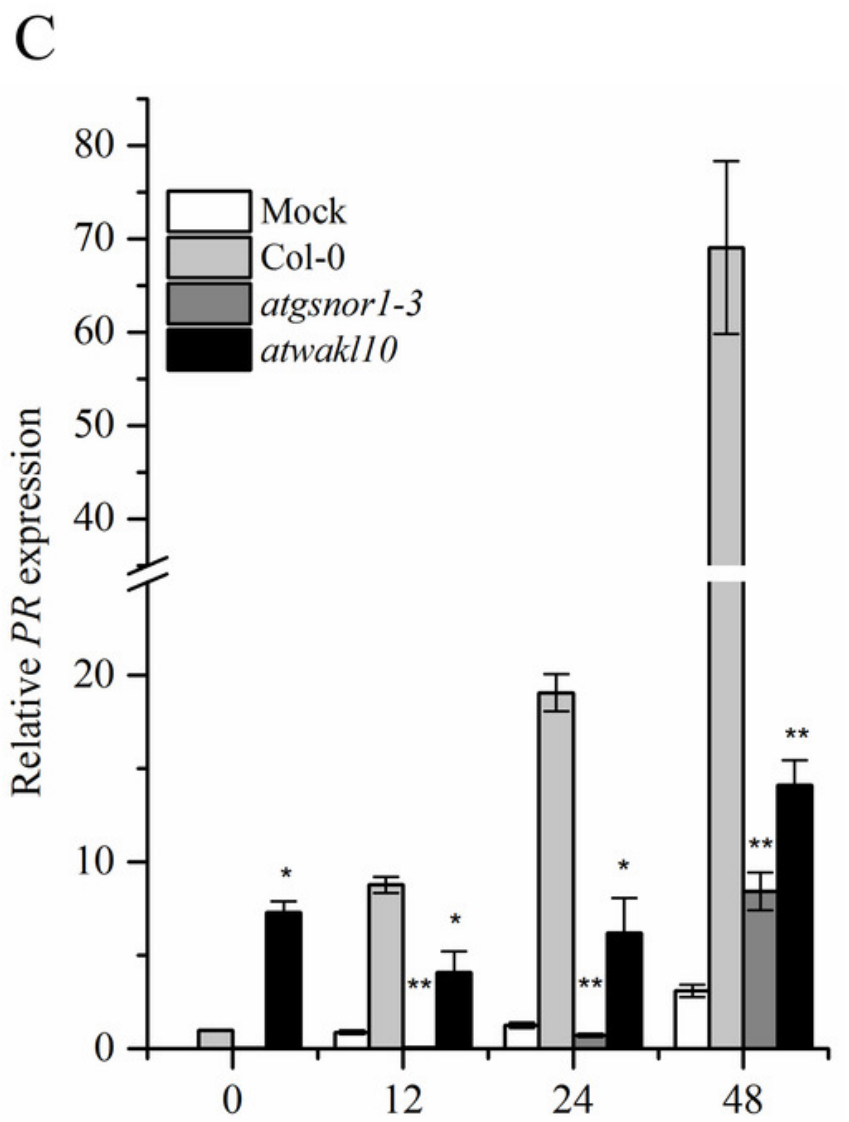

Time post inoculation (h) 


\section{Figure 3}

AtWALK10 positively regulates effector-triggered immunity

(A) Trypan blue staining showing HR response. (B) Pathogen growth determined as log colony forming unit (CFU) (C) Diaminobenzidine (DAB) staining depicting $\mathrm{H}_{2} \mathrm{O}_{2}$ accumulation and (D) cell death-induced electrolyte leakage after Pst DC3000 avrB challenge. The background in A and $C$ are modified for more clarity. The data points in $B$ and $D$ are the mean of three replicates. The pathogenicity experiment was repeated about 3 times while that of electrolyte leakage was repeated twice with similar results. Error bars represent $\pm \operatorname{SE}(n=3)$ while significant differences compared to WT are marked with asterisks [Student's t-test with $95(*)$ and $99 \%(* *)$ confidence levels]. 


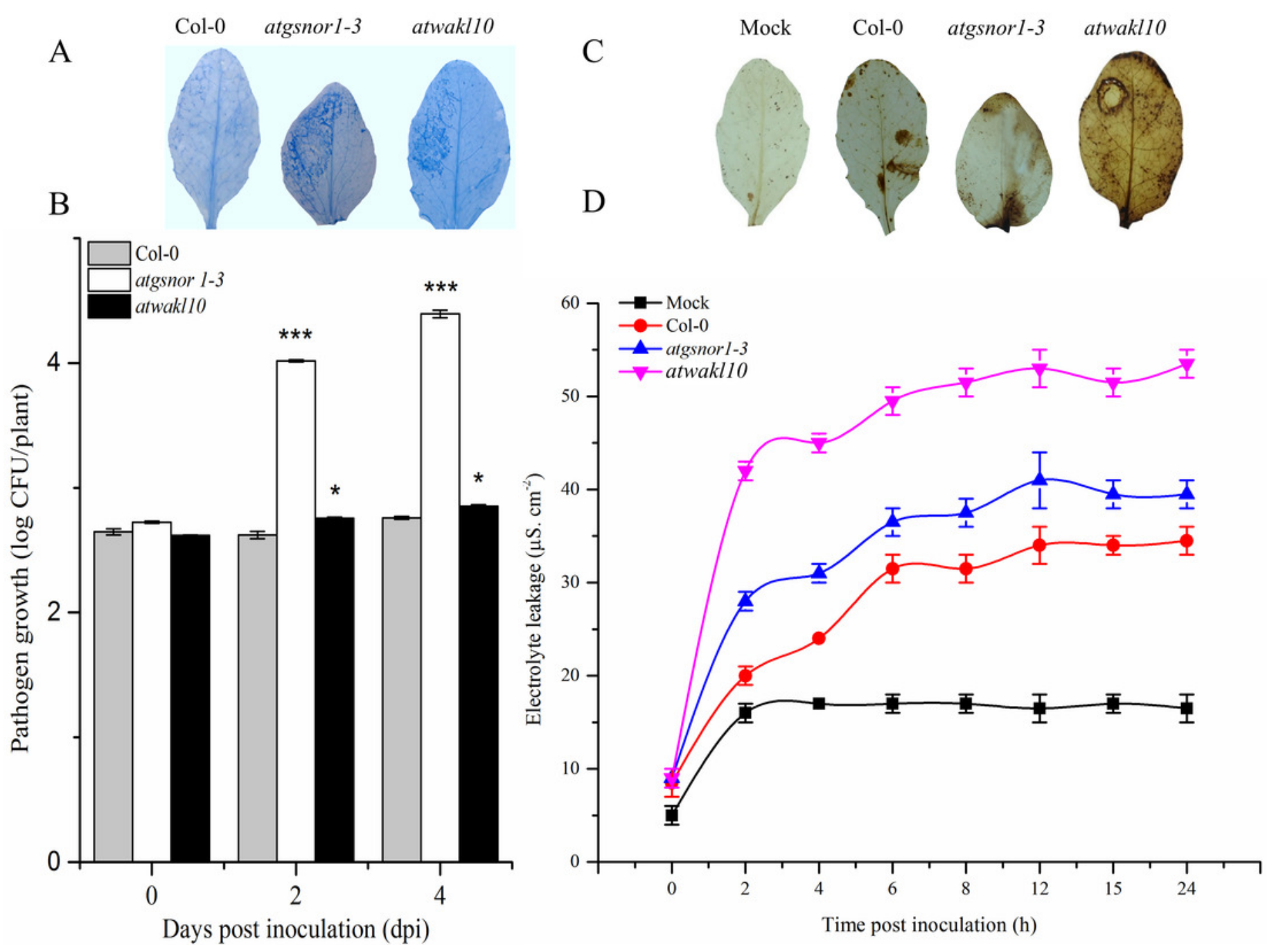


Figure 4

\section{Promoter analysis of AtWALK10}

(A) Genetic structure of AtWALK10. Green bars represent exons, while gaps between two exonic regions are introns. (B) Position and visualization of different transcription factor binding site (TFBS) $1 \mathrm{~Kb}$ upstream of transcription initiation site and (C) comparison of conserved regions of AtWALK10 and its orthologs in rice.
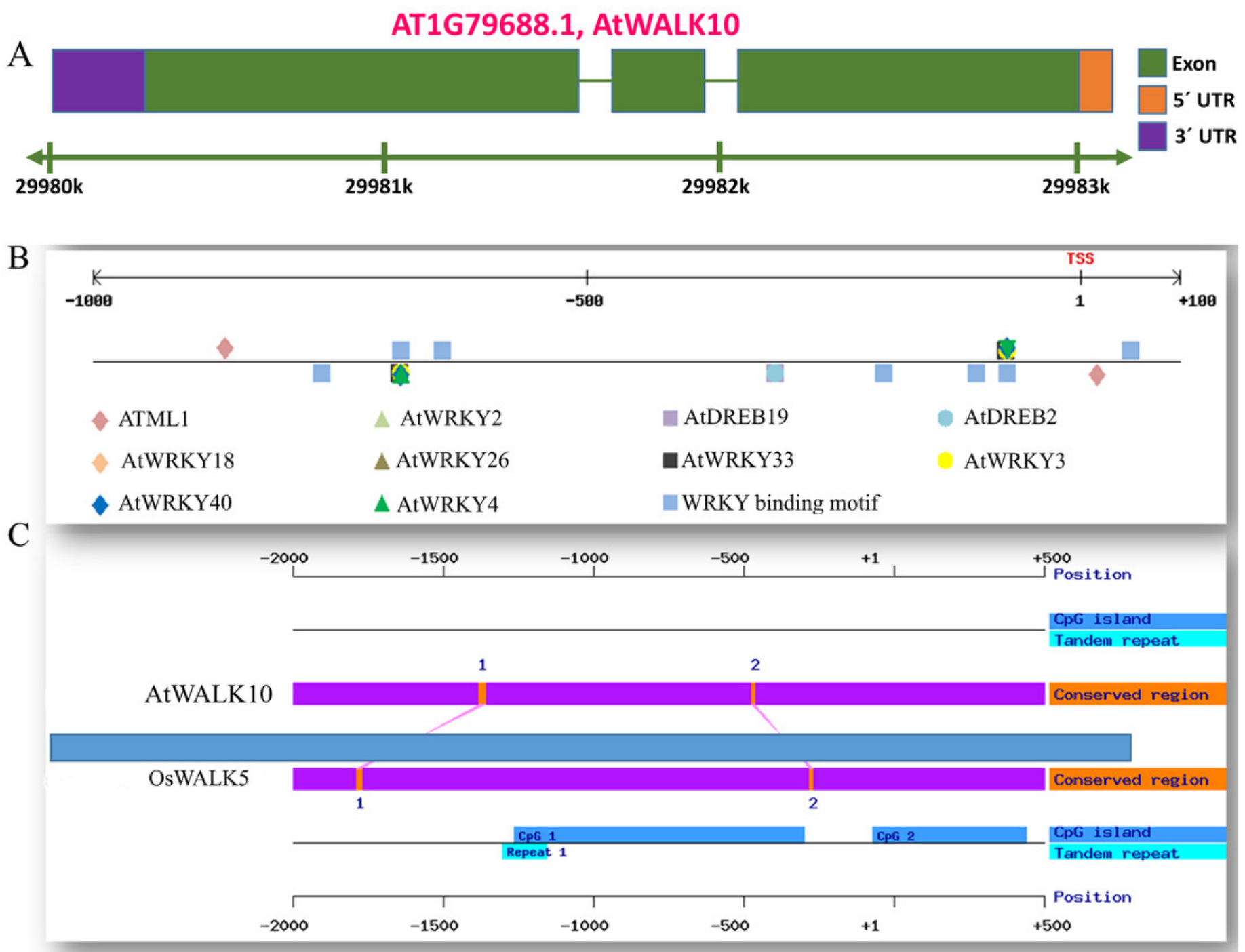


\section{Figure 5}

atwalk10 plants showed reduce germination percentage under high salinity conditions

(A) Germination of atwakl10 and WT plants on different salt conditions. (B) Germination

percentage in WT and mutant line after exposure to different salinity conditions. The data in the graph represents the mean of three replicates. The experiment was repeated three times with similar results. Error bars represents $\pm S E(n=3)$. 

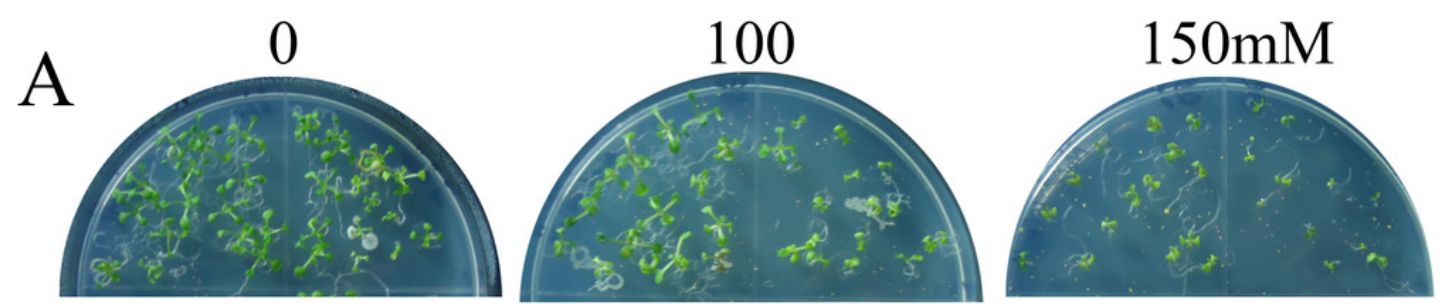

B

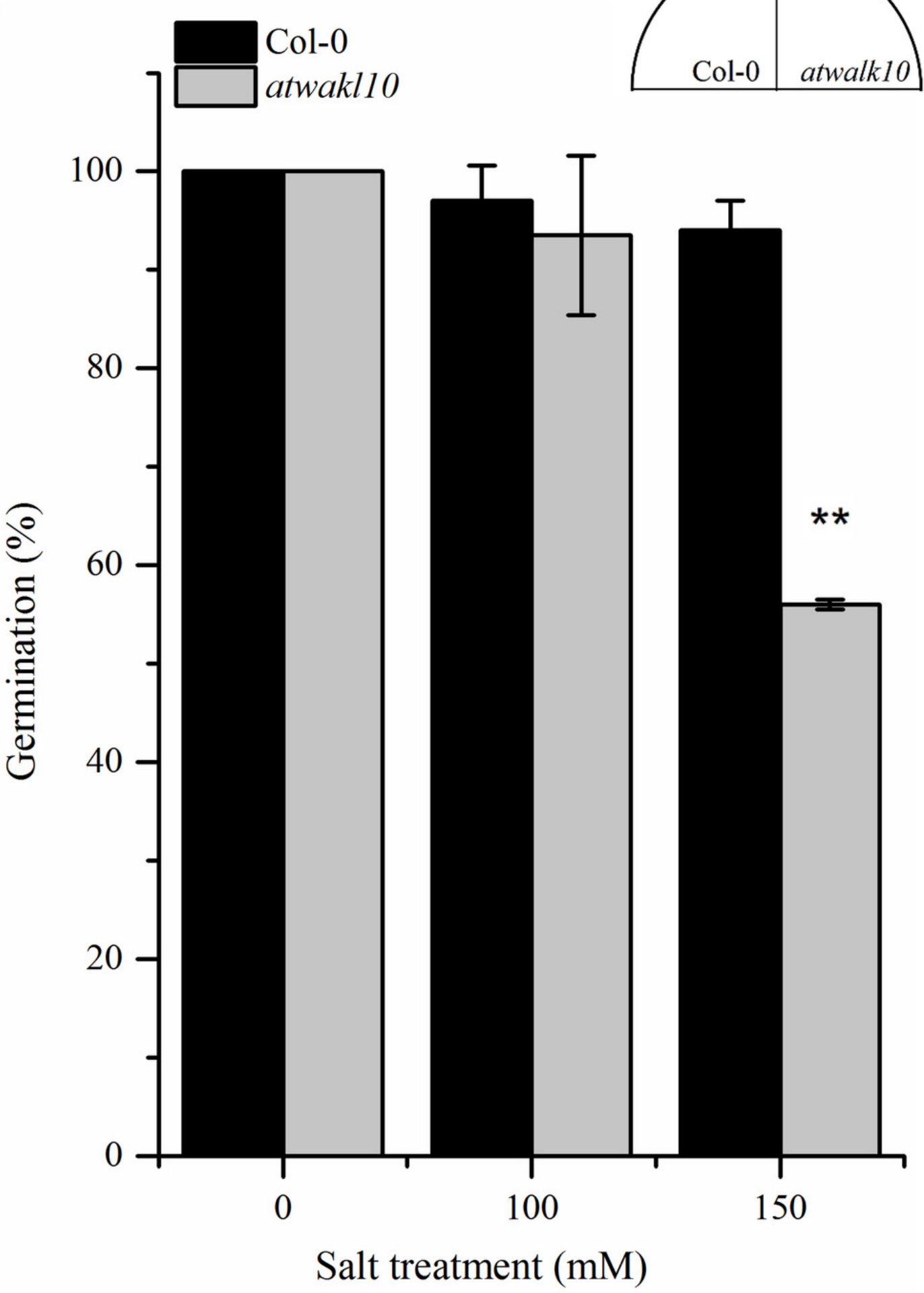




\section{Figure 6}

atwalk10 plants exhibit drought tolerance

(A) Phenotypes of indicated genotypes 0 days post-drought (after withholding water). (B) After 2 weeks of drought stress. (C) Phenotypes of plants after 2 weeks of drought stress, followed by re-watering. (D) Quantification of recovery rates after re-watering. The experiment was repeated twice with similar results.

A

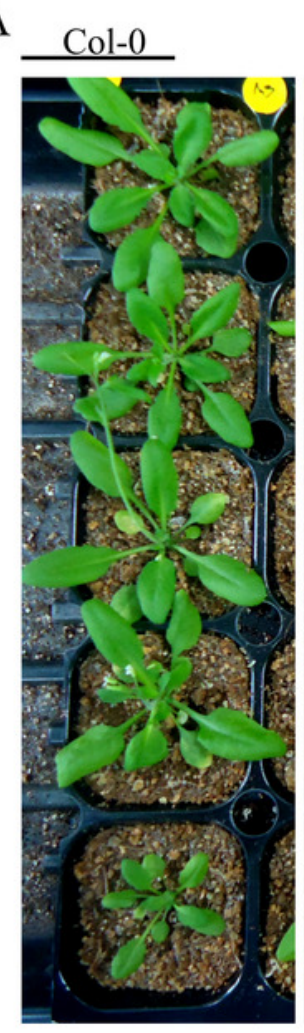

B

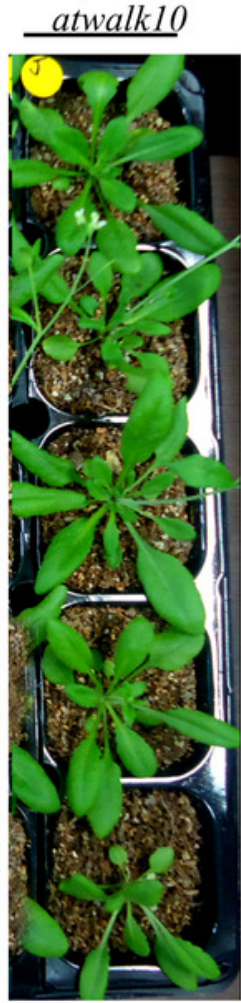

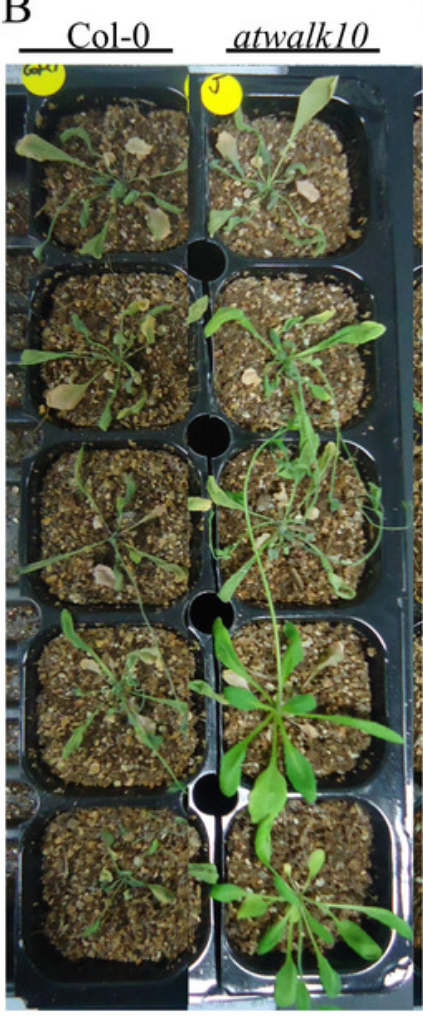

$\mathrm{C}$

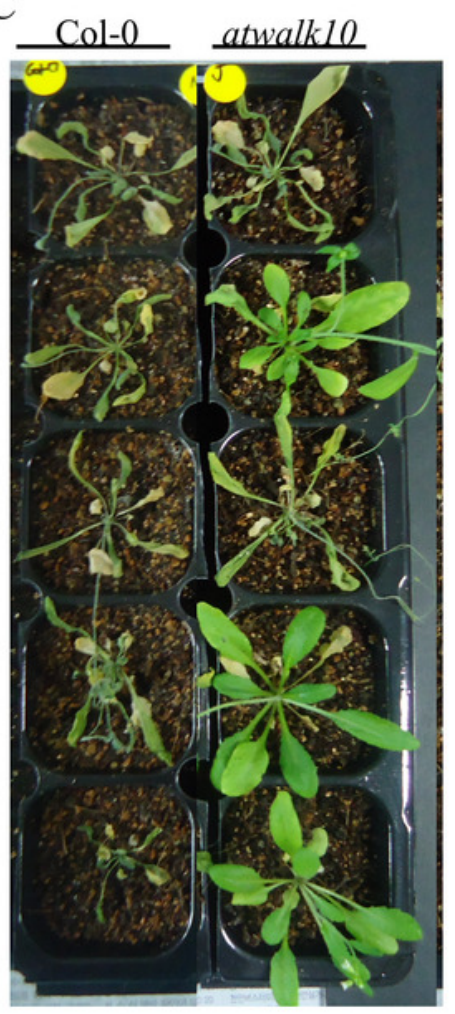

$\mathrm{D}$

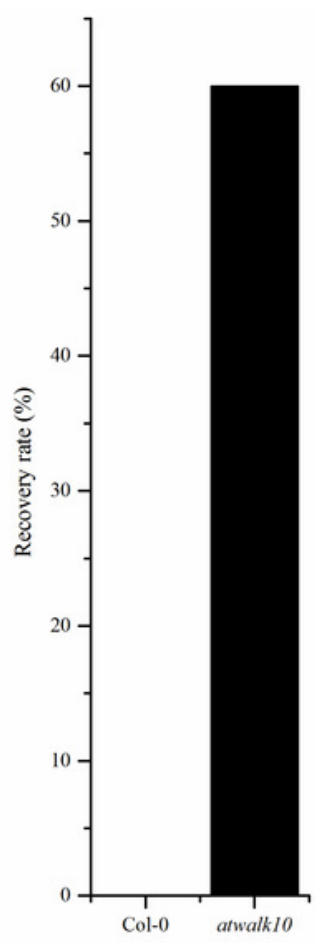




\section{Table $\mathbf{1}$ (on next page)}

List of Primers used in the study 
1 Table 1. List of Primers used in the study

\begin{tabular}{|l|l|l|l|}
\hline Gene Name & Acc No & Forward $\left(5^{\prime}-3^{\prime}\right)$ & Reverse $\left(5^{\prime}-3^{\prime}\right)$ \\
\hline Actin & AT3G18780 & GCTGGACGTGACCTTACTGA & CCATCTCCTGCTCGTAGTCA \\
\hline PR1 & AT2G14610 & GTGCAATGGAGTTTGTGGTC & TCACATAATTCCCACGAGGA \\
\hline AtWALK10 & At1g79680 & CACTAGCGCATGAACATGTTG & AAACCCGTCTCTGCTTTTAGC \\
\hline $\begin{array}{l}\text { Sail border } \\
\text { primer }\end{array}$ & SAIL_LB1 & $\begin{array}{l}\text { GCCTTTTCAGAAATGGATAAATAG } \\
\text { CCTTGCTTCC }\end{array}$ & \\
\hline
\end{tabular}

2

3 
